\title{
Anticipatory anxiety promotes satisficing during multi-cue probabilistic decision making
}

\author{
Hanna Oh-Descher, Hitomi Tanaka, Kevin S. LaBar \\ Duke University \\ Silvia Ferrari \\ Cornell University \\ Marc A. Sommer, Tobias Egner \\ Duke University
}

\begin{abstract}
Author Note
Hanna Oh-Descher, Department of Psychology and Neuroscience, and Center for Cognitive Neuroscience, Duke University; Hitomi Tanaka, Trinity College of Arts and Sciences, Duke University; Kevin S. LaBar, Department of Psychology and Neuroscience, and Center for Cognitive Neuroscience, Duke University; Marc A. Sommer, Department of Biomedical Engineering, Department of Neurobiology, and Center for Cognitive Neuroscience, Duke University; Silvia Ferrari, Department of Mechanical and Aerospace Engineering, Cornell University; Tobias Egner, Department of Psychology and Neuroscience, and Center for Cognitive Neuroscience, Duke University.

This work was funded by Office of Naval Research grant ONR N000141310561.

Correspondence concerning this article should be addressed to Tobias Egner, Department of Psychology and Neuroscience, and Center for Cognitive Neuroscience, Duke University, Levine Science Research Center, Box 90999, Durham, NC 27708. E-mail: tobias.egner@duke.edu
\end{abstract}




\begin{abstract}
Critical real-life choices involve making complex decisions in the presence of potential threats, for instance, in medical or military emergencies. Effective choices require a decision maker to efficiently weigh and combine multiple sources of uncertain information. As anxiety can disrupt cognitive performance, complex decision-making under uncertainty may be particularly compromised by potential threat. One way people overcome such cognitive limitations is to "satisfice" by selectively evaluating a subset of available information to quickly identify a goodenough, feasible solution. How satisficing decision-making plays out under anxiety, however, remains elusive. Here, we examined how healthy participants solve a multi-cue probabilistic classification task under anticipatory anxiety induced via a threat-of-shock manipulation. Specifically, we investigated individual differences in information (cue) usage based on participants' physiological responsiveness to threat, quantified by changes in skin conductance levels. In the absence of threat, all participants performed near-optimally, appropriately weighing and integrating all available cue information to guide their choices. Under threat-of-shock, however, participants who displayed high levels of anxiety employed a satisficing heuristic by ignoring the least important cue from their decision process, a strategy that uses less cognitive resources without sacrificing much accuracy. Moreover, anticipatory anxiety uncoupled the actual cue usage from explicit task knowledge. Taken together, these results suggest that, to cope with high levels of anticipatory anxiety, people satisfice by prioritizing high-value information to achieve fast and good-enough solutions.
\end{abstract}

Keywords: decision-making, multi-cue integration, satisficing, threat-of-shock, anticipatory anxiety 


\section{Introduction}

Real-life decision-making is computationally complex, as it requires efficiently evaluating and combining relevant pieces of information, each of which is predictive of an outcome with varying degrees of uncertainty. While an ability to effectively make such complex decisions under threat, such as in military combat or other high-stakes scenarios, is particularly critical for survival, it may also be quite challenging, because anxiety about potential impending negative events may compromise the capacity for optimal decision-making (for reviews, see Grupe \& Nitschke, 2013; Robinson, Vytal, Cornwell, \& Grillon, 2013). One way that people overcome cognitive limitations in complex decision-making is to rely on satisficing decision heuristics that selectively evaluate a subset of available information to quickly identify a goodenough, feasible solution (Simon, 1955, 1990). Surprisingly little is known, however, about the satisficing strategies that people may use under anticipatory anxiety. The goal of the present study is to characterize the manner in which complex decisions involving multiple probabilistic pieces of information are influenced by anticipatory anxiety induced by threat-of-shock. Anticipating a negative event induces a state of anxiety that can influence a large array of cognitive abilities (Robinson, Vytal, et al., 2013). The threat-of-shock paradigm is a robust, wellestablished technique, in which infrequent, randomly occurring electrical shocks induce sustained anxiety in healthy participants (Schmitz \& Grillon, 2012). Here, we specifically focused on task non-contingent, unpredictable, and uncontrollable threats, which trigger an anticipatory anxiety response characterized by an increase in skin conductance level (SCL) (Rhudy \& Meagher, 2000) and resemble free-floating anxiety in real-world contexts. Such taskunrelated anxiety can result in both detrimental and facilitating effects on a broad range of executive functions such as attentional control (e.g., Eysenck, Derakshan, Santos, \& Calvo, 
2007; Hu, Bauer, Padmala, \& Pessoa, 2012), working memory (e.g., Shackman et al., 2006;

Vytal,

Cornwell, Letkiewicz, Arkin, \& Grillon, 2013), and decision-making (e.g., Clark et al., 2012;

Keinan, 1987). Importantly, in line with predictions from the satisficing decision theory (Simon, 1955, 1990), Easterbrook (1959) proposed that behavioral changes observed under anxiety are driven by "attentional narrowing", which reduces the range of cue utilization and thereby enhances focus on the most important task-relevant cues. This attentional narrowing effect has often been observed through enhanced early perceptual processing of salient cues (e.g., Cain, Dunsmoor, LaBar, \& Mitroff, 2011; Cornwell et al., 2007), although the extension of this effect to subsequent decision-making processes remains unclear (cf., Shackman, Maxwell, McMenamin, Greischar, \& Davidson, 2011). Additionally, whether selective cue processing under anxiety is executed in an adaptive manner - a core characteristic of satisficing - via facilitating cue evaluation in order of their importance during decision-making is currently unknown.

The influence of anxiety on probabilistic decision-making has been rarely examined, but prior reports based on acute stress induced by the cold pressor test offer some useful insights. During a two-step reinforcement learning task, stress attenuated the contribution of model-based, goaldirected learning but not model-free, habitual decision-making (Otto, Raio, Chiang, Phelps, \& Daw, 2013). Similarly, during probabilistic classification learning, a pressor-induced stress group was more likely to engaged in a striatum-dependent habit learning strategy whereas the control group tended to adopt a hippocampus-dependent declarative strategy (Schwabe \& Wolf, 2012). Importantly, this difference was characterized behaviorally by a significant reduction in 
explicit task knowledge under stress without any observable difference in overall classification accuracy, suggesting that traditional decision performance measures (i.e., accuracy or response time) may not be strongly reflective of the underlying changes in decision strategy. Taken together, consistent with the multiple memory systems theory (Knowlton, Mangels, \& Squire, 1996; Poldrack et al., 2001), anxiety may shift decision-making to the habitual system to facilitate decision processes without sacrificing overall performance, which is often reflected by reduction of explicit task knowledge (for a review, see Schwabe \& Wolf, 2013).

To examine the effect of anticipatory anxiety on choice behavior, we employed a multicue probabilistic classification task (Oh et al., 2016), which allowed us to track and quantify participants' information (cue) usage during decision-making. Specifically, we primarily based our analyses on variational Bayesian inference, which yields better sensitivity in characterizing participants' implicit decision strategies than traditional performance metrics. In a previous study that examined decisions under time pressure, we demonstrated the engagement of a "droptheworst" satisficing strategy under high time pressure (Oh et al., 2016). That is, participants strategically ignored the least important cue(s) and considered only a subset of the most predictive information in their decision process. To investigate whether this adaptive shift to satisficing decision-making generalizes to decisions under anticipatory anxiety, or whether other satisficing strategies emerge under these conditions, we combined the multi-cue probabilistic classification task with the threat-of-shock paradigm, while recording participants' SCL throughout the experiment. This experimental manipulation allowed us to detect and compare strategies utilized during neutral versus threat-of-shock conditions, both at the within- and between-subjects level, as a function of individual differences in the effectiveness of the threat manipulation as quantified by SCLs. We predicted that anticipatory anxiety would (1) trigger 
shifts in strategy, promoting the use of satisficing heuristics under high arousal, and (2) induce reduction in declarative knowledge of the task-related information.

\section{Method}

\section{Participants}

Sixty right-handed individuals from the Duke University community participated in return for course credits. Since we planned to investigate individual differences based on subgrouping of participants by SCL response, we targeted the sample size of 20 participants/group based on previous studies on the effect of threat-of-shock on decision-making (e.g., Hu et al., 2012; Robinson, Overstreet, Charney, Vytal, \& Grillon, 2013). Three participants were excluded from the analysis, two due to a lack of measurable skin conductance responses to shock and one due to an equipment malfunction, leaving 57 participants $(38$ females; mean age $=$ 19.1 years; range $=18-21$ years). All participants provided informed consent in accordance with Duke University Institutional Review Board guidelines and reported no history of neurological or psychiatric illness, and no current psychoactive medication use.

\section{Stimuli}

The task stimuli consisted of 16 unique visual compound cues composed of four different features (cue dimensions: color, shape, contour, and line orientation), each of which was comprised of two sub-features (cue states: blue/red, circle/square, white/black, vertical/horizontal) (Fig. 1A). Each cue state was associated with a fixed weight (cue weights) predictive of the probability of "winning" (Table 1, Eqn. 1). The cue weights within a given cue dimension were complementary and summed to one. The net weights, which signify the relative 
importance of an individual cue dimension in predicting the winning stimulus, were defined by the difference between the cue weights comprising each dimension. Thus, a cue dimension for which one cue state has weight 0.9 and the other has weight 0.1 provides more information for predicting the winning stimulus than a cue dimension with states of weights 0.6 and 0.4 . The assigned cue weights created a compensatory environment, in which the highest weighted cue, $c_{1}$, can be out-weighted by some combinations of $c_{2}, c_{3}$, and $c_{4}$. To eliminate the possibility that differences in physical cue saliency might affect choice behavior, the weights were randomly assigned to the different cue dimensions for each participant and stayed fixed throughout the experiment.

In the threat-of-shock phase (see Procedure), five shock stimuli were administered to the right wrist for $6 \mathrm{~ms}$. Shock intensity was calibrated for each participant using an ascending staircase procedure to be perceived as "highly annoying but not painful" $(M=32.8 \mathrm{~V}, S D=8.4$; Dunsmoor, Mitroff, \& LaBar, 2009). Throughout all phases of the experiment, SCL was measured with $\mathrm{Ag}-\mathrm{AgCl}$ electrodes placed on the middle phalanx of the second and third digits of the left hand. Shock delivery and SCL were recorded using a MP-150 system connected to AcqKnowledge software (BIOPAC Systems, Goleta, CA).

\section{Multi-cue probabilistic classification task}

Participants performed a multi-cue probabilistic classification task (Oh et al., 2016), where on each trial they were presented with two different stimuli and were asked to predict the stimulus that is more likely to "win" (Fig. 1A). By sampling from the full set of 16 compound cues, we used 120 unique stimulus pairs, which could differ anywhere from one to four cue dimensions (Fig. 1C). This difference determined the underlying winning probabilities, which in 
turn governed feedback. That is, the probability that a left $(L)$ or a right $(R)$ stimulus would win was based on the cue states comprising the left stimulus, $C_{L}=\left\{c_{L, 1}, C_{L, 2}, C_{L, 3}, C_{L, 4}\right\}$, and the right stimulus, $C_{R}=\left\{C_{R, 1}, C_{R, 2}, C_{R, 3}, C_{R, 4}\right\}$, and their associated weights, $W_{L}=\left\{w_{c L, 1}, W_{c L, 2}, W_{c L, 2}, W_{c L, 4}\right\}$ and $W_{R}=$ $\left\{w_{c R, 1}, W_{c R, 2}, W_{c R, 2}, W_{c R, 4}\right\}$ (Table 1). More precisely, the sum of cue weight difference between the stimulus pair determined the winning probability given the cue states, $C_{L}$ and $C_{R}$ :

$$
\begin{gathered}
P\left(L \mid C_{L}, C_{R}\right)=\frac{10^{\sum_{i=1}^{4}(w c L, i-w c R, i)}}{\left.1+10^{\sum_{4 i=1}(w c L, i-w c R, i}\right)} \\
P\left(R \mid C_{L}, C_{R}\right)=1-P\left(L \mid C_{L}, C_{R}\right)
\end{gathered}
$$

where $i$ represents cue dimension. Based on Eqns. 1 and 2, the winning stimulus was determined probabilistically on a trial-by-trial basis, and the feedback was provided on-screen via the words "win" or "lose", displayed upon each choice response.

\section{Procedure}

Prior to the experiment, participants completed both State and Trait parts of the StateTrait Anxiety Inventory (STAI; Spielberger, 1983). The multi-cue probabilistic classification task was divided into three phases: an initial learning (IL) phase, followed by a neutral (no-shock, NS) phase, and a threat-of-shock (TS) phase. Each phase consisted of 240 trials, which were randomized and grouped into 4 blocks of 60 trials per phase, with short breaks in between. Specifically, Blocks 1 and 2 were comprised of the full set of all 120 possible stimulus pairs, followed by Blocks 3 and 4, which consisted of the mirror images of the first set. On each trial, participants were presented with a stimulus pair and asked to indicate their choice via keyboard 
button press using their right index (left stimulus) and middle (right stimulus) fingers, within a 2 s response window. Once a valid response was made, feedback was presented for $500 \mathrm{~ms}$, which was followed by a $1 \mathrm{~s}$ inter-trial-interval.

Enhanced startle responses can be triggered by placement of shock electrodes on participants' wrist alone (Grillon \& Ameli, 1998), indicating that this procedure, even in the absence of threat, could induce a general increase in baseline anxiety. To avoid this kind of spillover effect of the shock condition contaminating decision behavior in neutral blocks, the treat-of-shock manipulation was introduced to participants only upon completion of the IL and NS phases, when we performed the shock intensity calibration. Then, subjects were informed that a few shocks would be delivered throughout the phase, independent of their performance. During the TS phase, five shock trials were included at random time points, in addition to 240 valid trials, with a maximum of two shocks per block. Shocks were administered at the time of feedback along with a visual shock symbol (Fig. 1B), and these trials were excluded from data analyses.

After finishing the TS phase, participants completed a post-experimental survey assessing their explicit task knowledge. First, participants were asked to rank cue dimensions from the most to the least informative. Then they indicated the number of cues, on average, they thought they had taken into account before making decisions in the NS and TS phases. Finally, to probe their knowledge on cue states, they were presented with four different compound stimulus pairs varying in only one cue dimension (e.g., shape). For each pair, participants were asked to choose a stimulus with a higher probability of winning and indicate how likely they will win the given trial along a range from $50 \%$ to $100 \%$. 


\section{Skin conductance level (SCL) analysis}

SCLs were calculated as the mean response over each block using the Autonomate toolbox (Green, Kragel, Fecteau, \& LaBar, 2014). To minimize the influence of shock-triggered responses on SCLs, time epochs containing shock delivery and three trials following the shock $(\sim$ $8 \mathrm{~s}$ ) were removed from the analysis. For each participant, SCLs were range-corrected based on subject-specific minimum and maximum SCLs observed during the experiment to account for individual differences (Lykken \& Venables, 1971), and averaged across blocks per phase. Then, changes in SCL between the NS and TS phases were used $(\triangle S C L=S C L(T S)-S C L(N S))$ to quantify the effect of threat-of-shock in inducing anticipatory anxiety.

\section{Participant clustering}

To characterize how anticipatory anxiety differentially influenced decision strategies employed during the TS phase, we partitioned participants based on $\triangle S C L$. Specifically, participants were clustered into low $(n=14)$, mid $(n=20)$, and high $(n=23)$ responders using the Ckmeans.1d.dp algorithm (Wang \& Song, 2011). This algorithm yields optimal onedimensional clustering by minimizing within-cluster distances from each data point to its corresponding cluster mean, and therefore, provides an objective means of reliable and natural grouping of participants. Note, however, that using a tercile split does not change the overall results we report.

\section{Behavioral performance analysis}

Performance data analyses were based on correct choices favored by the probability of winning (for additional details, see Oh et al., 2016). In other words, whenever a participant chose 
a stimulus with a higher probability of winning defined by equations (1) and (2), the trial was considered correct even though the participant may have experienced negative feedback due to the probabilistic nature of the feedback. In evaluating decision accuracy (\% correct choices), trials with two stimuli that had an equal sum of weights were excluded since a correct choice cannot be defined, i.e., $P\left(L \mid C_{L}, C_{R}\right)=P\left(R \mid C_{L}, C_{R}\right)=0.5$. In reporting t-test results, degrees of freedom were corrected for unequal variance where necessary. Similarly, in reporting analysis of variance (ANOVA) measures, violations of sphericity assumptions were corrected by Greenhouse-Geisser correction.

\section{Decision strategy model selection}

To identify decision strategies that individual participants may have applied to solve the task, we explored a large set of plausible decision models, accounting for every possible combination of cue dimension usage (Fig. 3A). Model Opt represented an optimal cueintegration model where participants integrate all four cue dimensions in making their choices, whereas Models 1 through 14 represented various combinations of sub-optimal cue integration. The expected accuracy was estimated under the assumption that a decision maker has learned to make optimal choices using a given set of cues included in each strategy model (Fig. 3B). Since relatively uninformative cue dimensions, such as $c_{3}$ and $c_{4}$, do not strongly influence final outcomes, the expected accuracy of some sub-optimal strategy models is close to optimal. Given the probabilistic nature of the task, human participants are prone to have higher decision noise and to make more errors than the ideal observer, which could make the performance outcomes of these sub-optimal and the optimal models indistinguishable. Therefore, to achieve better sensitivity, we performed model comparison using variational Bayesian inference (Drugowitsch, 
2013) based on the decision strategy models. For a given model $m(m=1, \ldots, 14$, opt $)$, we constructed an $N$ (total number of trials) $\times n_{m}$ (number of cue dimensions considered in model $m$ ) input matrix, $x_{m}$, coding for the presence of cue dimension differences on each trial, i.e., 0 for $w_{c L, i}=w_{c R, i}, 1$ for $w_{c L, i}>w_{c R, i}$, and -1 for $w_{c L, i}<w_{c R, i}$ for a cue dimension $i$. This input matrix, $x_{m}$, was used to fit a logistic model, which returned a lower bound of the marginalized log-likelihood, $P\left(D \mid\right.$ Model $\left._{m}\right)$, of the observed choice data, $D$ :

$$
\begin{gathered}
P\left(\text { choice }=L \mid x_{m}, w_{m}\right)=1 \\
P\left(w_{m} \mid \alpha\right)=\mathcal{N}\left(w_{m} \mid 0, \alpha^{-1} I\right)(4) P(\alpha)=\operatorname{Gamma}\left(\alpha \mid a_{0}, b_{0}\right)(5)
\end{gathered}
$$

with $w_{0}$ representing a $N \times 1$ vector for estimating the intercept. We obtained the hyper priors, $a_{0}$ and $b_{0}$, based on the mean $(\mu)$ and the variance $\left(\sigma^{2}\right)$ of the observed distribution of subjective cue weights in the IL phase, according to an empirical Bayesian approach:

$$
a_{0}=\mu \sigma \_22, b 0=\sigma \_\mu 2
$$

which resulted in $a_{0}=0.55$ and $b_{0}=0.79$. Changing these values to correspond to relatively uninformative hyper priors $\left(a_{0}=0.01\right.$ and $\left.b_{0}=0.0001\right)$ did not change the overall results of model comparison.

To summarize the model comparison results at the group level, we employed a Bayesian model selection procedure to fit the hierarchical model, where models are treated as random effects that could vary across subjects (Rigoux, Stephan, Friston, \& Daunizeau, 2014; Stephan, Penny, Daunizeau, Moran, \& Friston, 2009). To rule out the possibility that differences in observed model frequencies could be driven by chance, we calculated protected exceedance probabilities and associated Bayesian omnibus risks (BORs). The protected exceedance probability reflects 
the belief that a model, $m$, provides a more likely explanation of the data than any other model, beyond chance, given the marginalized likelihoods obtained from the variational Bayesian inference procedure above. The BOR evaluates the probability that the observed difference in model frequencies occurred by chance, i.e., the null-hypothesis whereby all model frequencies are equal. Hence, BOR can be considered as analogous to a classical pvalue. The Bayesian model selection results reported here were calculated using the spm_BMS routine of the SPM12 software suite (http://www.fil.ion.ucl.ac.uk/spm/software/spm12/).

\section{Explicit knowledge of the cue structure}

Post-experimental survey responses were analyzed to examine participants' explicit knowledge about the cue structure and strategies employed in each phase. Spearman's rank-order correlation was used to quantify explicit knowledge by comparing each participant's survey rating with assigned weights, $w_{n e t . i}(i=1, \ldots, 4$; Table 1$)$, as well as inferred subjective cue weights, $w_{\text {opt }}$ (Eqn. 3). The correlation between the survey rating and assigned weights, $r_{w}$, describes how well participants learned the true underlying cue structure. More importantly, the correlations between the survey rating and subjective cue weights, $r_{s w, N S}$ and $r_{s w, T S}$, indicate the degree of correspondence between participants' explicit knowledge of the cue structure versus the actual cue dimension weighting employed in the NS and TS phases. For further analyses, all correlation coefficients were Fisher z-transformed to achieve normal distribution.

To further examine the relationship between participants' cue usage and their explicit knowledge across conditions, we compared z-transformed correlation coefficients between the NS and TS phases. Specifically, the concordance between actual strategy and declarative knowledge was measured by changes in subjective cue weights from the NS to TS phase in 
relation to participants' explicit survey ratings $\left(\Delta r_{s w}=z\left(r_{s w, T S}\right)-z\left(r_{s w, N S}\right)\right)$. Hence, a positive $\Delta r_{s w}$ indicates a greater concordance between participants' applied cue weighting and their expressed explicit knowledge in the TS compared to the NS phase. Conversely, a negative $\Delta r_{s w}$ suggests an increased mismatch between the cue usage and survey rating in the TS phase. Based on previous studies that demonstrated a reduction in the use of declarative task knowledge under stress (e.g., Schwabe \& Wolf, 2012), we hypothesized that the participants with a greater increase in arousal $(\triangle S C L)$ would show a greater dissociation between declared and applied task knowledge in the TS relative to the NS phase. To investigate this relationship, we performed a quadratic regression with $\triangle S C L$ as the independent variable and $\Delta r_{S w}$ as the dependent variable:

$$
\Delta r_{s w}=\beta_{0}+\beta_{1} \times \Delta S C L+\beta_{2} \times \Delta S C L^{2}+\varepsilon
$$

where $\beta_{1}$ and $\beta_{2}$ represent regression coefficients, $\beta_{0}$ is a constant, and $\varepsilon$ is a random error. In addition to reporting adjusted $R^{2}\left(R_{a d j}{ }^{2} \quad\right)$, significance of the quadratic regression was tested via ANOVA.

\section{Results}

\section{Physiological response to threat-of-shock}

At the population level, analysis of SCLs revealed a main effect of phase, $F(2,112)=$ 29.43, $\eta_{p}^{2}=0.35, p<0.001$. Post-hoc pairwise comparisons using Bonferroni correction showed a significant increase of SCLs in the TS phase compared to the IL and NS phases ( $p s<$ $0.001)$, with no difference between the IL and NS phases $(p=0.213)$. To confirm that this selective increase in SCLs in the TS phase was not driven by any general trend for increased 
SCLs over time, we performed a 3 (phases) $\times 2$ (sets) repeated-measures ANOVA (Fig. 2A).

Each set consisted of 120 unique stimulus pairs and therefore, this approach additionally controlled for any variance in SCL caused by task difficulty. This analysis revealed a significant effect of set, $F(1,56)=5.29, \eta_{p}^{2}=0.09, p=0.03$, which was due to an overall decrease rather than increase in amplitude with time. Taken together, these results confirm that the threatofshock manipulation successfully enhanced autonomic arousal.

To examine how autonomic arousal responses to the threat-of-shock manipulation varied across individuals, and how this responsivity may modulate decision strategies, we divided participants into low $(M=-0.13, S D=0.08)$, mid $(M=0.10, S D=0.07)$, and high $(M=$ $0.39, S D=0.09)$ responders based on differences in SCLs between the TS and NS phases ( $\triangle \mathrm{SCL}$ ) via the Ckmeans.1d.dp clustering algorithm (Wang \& Song, 2011) (Fig. 2B). As expected, one-way ANOVA revealed a significant difference in $\triangle \mathrm{SCLs}$ between the three groups, $F(2,54)=198.01, \eta_{p}^{2}=0.88, p<0.001$, suggesting a reliable clustering of participants based on their level of autonomic arousal under threat-of-shock.

Lastly, we sought to examine the relationship between participants' STAI scores and changes in SCLs throughout the experiment. Neither state $(M=34.28, S D=8.37)$ nor trait $(M=38.35, S D=10.04)$ anxiety scores differed across the three groups (one-way ANOVA, $p s>0.5)$. Interestingly, trait anxiety scores were significantly correlated with SCLs in the IL $(r=0.26, p=0.049)$ and TS $(r=0.29, p=0.027)$ phases but not in the NS $(r=0.12, p=$ 0.390) phase, suggesting that participants with high trait anxiety displayed enhanced arousal at the beginning of the experiment as well as under the threat-of-shock condition. No significant correlations were found with state anxiety scores, nor between STAI scores and $\triangle$ SCLs. 


\section{Task performance}

Task performance was assessed based on the correct decision defined by the sum of cue weights, independent of the feedback provided (see Methods). All three SCL groups learned the task reliably, gradually improving and stabilizing performance over the course of the experiment, which was demonstrated by a significant main effect of phase, $F(2,108)=30.85, \eta_{p^{2}}=$

0.36, $p<0.001$ (Fig. 2C). We observed no between-groups effect, $F(2,54)=0.52, \eta_{p}^{2}=$ $0.02, p=0.597$, nor a phase $\times$ group interaction, $F(4,108)=0.18, \eta_{p}^{2}=0.06, p=0.178$, indicating that all groups performed the task equally well. This observation was in line with the changes in RT throughout the experiment (Fig. 2D). RT decreased as participants become more familiar with the task, which was shown by a significant main effect of phase, $F(2,108)=$ 128.00, $\eta_{p}{ }^{2}=0.70, p<0.001$. Again, we observed no between-groups effect, $F(2,54)=1.30$, $\eta_{p}^{2}=0.05, p=0.282$, nor a phase $\times$ group interaction, $F(4,108)=2.25, \eta_{p}^{2}=0.08, p=$ 0.069 .

In sum, summary performance measures of mean RT and accuracy were not affected by responsiveness to threat-of-shock. However, these data cannot convey whether the underlying decision strategies producing these compound results may have been altered by the threatofshock manipulation, especially since the expected accuracy of some sub-optimal strategy models is near-optimal (Fig. 3B; see Method). Hence, to achieve better sensitivity, we employed variational Bayesian inference to examine how threat-of-shock may affect decision strategy. 


\section{Decision strategy model comparison}

We employed variational Bayesian inference to gauge participants' decision making strategies (Drugowitsch, 2013) and to quantify the most likely strategy model at the group level (Rigoux et al., 2014; Stephan et al., 2009). We explored 15 different plausible decision strategy models covering every possible case of cue usage, with Model Opt representing the optimal cueintegration model (Fig. 3A). The protected exceedance probabilities characterize how much more frequent a particular strategy model was employed than any other model, above and beyond chance, given the group model evidences, which are also reflected in BORs (Rigoux et al., 2014). In the NS phase, the optimal cue integration model, Model Opt, was the winning model for all three groups, with protected exceedance probabilities of $0.93(B O R=0.013$; Low, Fig. 3C), $0.96(B O R<0.001$; Mid, Fig. 3D), and $0.93(B O R<0.001$; High, Fig. 3E), respectively. The threat-of-shock manipulation did not have any effect on cue usage for the low and mid responder groups, for whom Model Opt remained the winning model with an exceedance probability of $0.96(B O R<0.001$; Fig. 3C) and $0.98(B O R<0.001$; Fig. 3D $)$. The most likely decision model for the high responder group in the TS phase, however, switched to Model 11, with a protected exceedance probability of $0.84(B O R<0.001$; Fig. 3E). This result indicates that participants who experienced the greatest increase in arousal under the threatofshock manipulation stopped using the weakest cue in their decision making process, instead relying on only the three most informative cues.

\section{Post-experimental survey}

In the post-experimental survey, participants were first asked to rank cue dimensions from the most to the least informative. Participants' ratings of cue dimensions showed a 
significant positive correlation with the assigned weights, wnet.i (mean $r_{w}=0.65, S E M=0.06$;

one-sample $t(56)=8.28, p<0.001,95 \% \mathrm{CI}[0.95,1.56])$, without any between-group difference, $F(2,54)=0.53, p=0.592$, suggesting that all three groups of participants were able to explicitly describe the relative importance of the true underlying cue weights correctly to some extent. Similarly, their survey cue ratings and the subjective weights, $w_{\text {opt }}$, showed significant positive correlations in both the NS (mean $r_{s w, N S}=0.57, S E M=0.06 ; t(56)=7.35, p<$ $0.001,95 \%$ CI $[0.78,1.36])$ and the TS (mean $r_{s w, T S}=0.63, S E M=0.06 ; t(56)=8.81, p<$ $0.001,95 \%$ CI $[0.89,1.42])$ phases. No difference was observed across the responder groups, $F(2,54)=1.60, \eta_{p}^{2}=0.06, p=0.211$, along with no main effect of phase, $F(1,54)=0.58$, $\eta_{p}^{2}=0.01, p=0.451$, nor phase $\times$ group interaction, $F(2,54)=0.53, \eta_{p}^{2}=0.02, p=$ 0.591.

To further investigate the relationship between participants' explicit knowledge and cue usage across phases, we assessed strategy concordance with declarative knowledge $\left(\Delta r_{s w}\right.$; see Methods), which reflects changes in subjective weights from the NS to TS phase relative to survey cue ratings. If participants formed more accurate insights to their cue usage or refined strategies according to their declarative knowledge of the cue structure as the experiment progressed, $\Delta r_{s w}$ would be positive. Conversely, if there was an increased discordance between participants' explicit knowledge and cue usage in the TS phase, $\Delta r_{s w}$ would be negative, indicating that subjects failed to employ their declarative knowledge of cue weights in executing decisions under the threat-of-shock condition. To examine the effect of arousal $(\triangle S C L)$ on strategy concordance $\left(\Delta r_{s w}\right)$, we fit a quadratic model (Eqn. 7), which revealed a significant inverted-U-shaped relationship, $\operatorname{Radj}^{2}=0.12, F(2,54)=4.92, p=0.011$ (Fig. 4). A simple linear regression did not show any meaningful relationship, $\operatorname{Radj}^{2} \quad=0.04, F(1,55)=1.97, p=$ 
0.166. These results suggest that participants who experienced moderate levels of arousal during the TS phase displayed enhanced concordance between their declarative knowledge and applied strategy, whereas those who experienced low or high levels of arousal showed increased discordance between their subjective cue weighting and explicit knowledge under the threatofshock. Additionally, the high responder group demonstrated a significantly negative correlation between $\Delta \mathrm{SCL}$ and $\Delta r_{s w}$ (Pearson's $r=-0.60, p=0.003$ ), which is in line with the possibility that this group deviated from using their internal model consistently when moving from the NS

to the TS phase. In other words, even though high SCL responders were able to articulate the relative utility of all four cues to a comparable extent as other participants after the experiment, during the TS phase they did not employ this knowledge consistently to guide their decisions.

Next, participants were asked the number of cue dimensions used in each phase. On average, subjects reported that they used 2.09 cues $(S E M=0.09)$ in the NS phase and 2.32 cues $(S E M=0.09)$ in the TS phase, which demonstrated a significant main effect of phase, $F(1,54)=4.86, \eta_{p}^{2}=0.08, p=0.032$, but without a phase $\times$ group interaction, $F(2,54)=$ $0.10, \eta_{p}^{2}=0.004, p=0.906$, nor a between-groups effect, $F(2,54)=1.56, \eta_{p}^{2}=0.06, p=$ 0.220. The discrepancy between results from the Bayesian strategy model selection and survey responses seems to suggest that a majority of participants did not have precise insight into the strategies (i.e., cue utilization) they employed to solve the task, which replicated previous experiments using this protocol (Oh et al., 2016). Lastly, subjects were asked to identify the cue state with the higher weight within each cue dimension (e.g., choice of blue vs. red). The mean number of correct responses was $3.63(S E M=0.08)$ out of 4 and there was no difference across 
responder groups, $F(2,54)=0.77, p=0.466$, indicating that all responder groups were able to identify the better cue state to a similar extent.

\section{Discussion}

To characterize the impact of anticipatory anxiety on complex decision-making, we tested healthy participants on a multi-cue probabilistic classification task under neutral and threat-of-shock conditions. Specifically, we focused on examining changes in post-learning decision performance, once participants had formed sufficient understanding of the cue structure of the task. To account for individual differences in susceptibility to the threat-of-shock manipulation, participants were clustered into three responder groups based on changes in SCLs from the NS to TS phase. In the neutral phase, all three groups of participants utilized information near-optimally, appropriately weighting and integrating all available cues to make choices. Under threat of shock, however, the high responder group adopted the drop-the-worst satisficing strategy by considering only the three most informative cues and ignoring the least important cue, whereas the low and mid responder groups did not show any change in strategy. Although suboptimal, this shift to the drop-the-worst heuristic was adaptive, since it yielded a comparable performance outcome while using less information. In addition, strategy concordance with participants' declarative knowledge exhibited an inverted-U relationship with $\triangle S C L$, suggesting that a moderate level of arousal can foster the formation and use of accurate, explicit strategy knowledge. This concordance, however, decreased with increasing arousal, indicating that participants who experienced greater anxiety demonstrated higher divergence of implicit strategy from their declarative cue knowledge. These findings suggest that high levels of 
anticipatory anxiety may trigger a shift from near-optimal to satisficing decision making, which is also associated with decreased implicit-explicit strategy concordance.

Adaptive satisficing is a form of bounded rationality that allows for good-enough decisionmaking when normatively optimal solutions are not feasible (Gigerenzer \& Goldstein, 1996; Simon, 1955), as in many real-world situations. Satisficing strategies depend heavily on exploiting the structure of the environment, thereby simplifying the decision problem without any significant impairment in performance (Gigerenzer \& Gaissmaier, 2011; Gigerenzer \& Goldstein, 1996; Simon, 1990). Although affect and emotion are thought to influence satisficing behaviors (Simon, 1990), only a handful of studies have systematically examined this relationship based on risky decision scenarios (e.g., Pachur, Hertwig, \& Wolkewitz, 2014), where the probabilities of possible outcomes were explicitly available to participants. While prominent theories have conjectured that anxiety reduces available cognitive resources and therefore restricts utilization of threat-irrelevant information (Easterbrook, 1959; Eysenck et al., 2007), the exact nature of this change during decision-making, especially under uncertainty, has remained unclear. Here, in accordance with this broad prediction, we demonstrated a reduction in low-information cue utilization under heightened anxiety. Importantly, this change in choice behavior was adaptive: to cope with anxiety, participants experiencing high arousal during the TS phase employed a satisficing heuristic that used less information without sacrificing overall performance. This observation is also consistent with our previous findings on satisficing under time pressure, where participants demonstrated adaptive cue discounting by systematically ignoring the least informative cue(s) with increasing time pressure (Oh et al., 2016). We here expanded these results by demonstrating that internally-induced pressure (i.e., anxiety) similarly fosters the switch to the drop-the-worst satisficing decision-making strategy. 
Much work in probabilistic decision-making suggests that acute stress fosters habitual learning and attenuates contributions of the goal-directed system (Otto et al., 2013; Schwabe \& Wolf, 2012; for reviews, see Phelps, Lempert, \& Sokol-Hessner, 2014; Schwabe \& Wolf, 2013). Although adopting either strategy often does not lead to a significant difference in probabilistic classification accuracy, the use of the habitual system is often linked to diminished explicit task knowledge (Foerde, Knowlton, \& Poldrack, 2006; Schwabe \& Wolf, 2012). A similar trend was observed for participants under emotional arousal (Thomas \& LaBar, 2008). That is, compared to a control group, participants in the emotional condition, where outcomes were paired with phobic stimuli (e.g., snake/spider), were more prone to using a simple, suboptimal strategy, with less declarative insights about probabilistic cue-outcome associations. Interestingly, a recent functional neuroimaging (fMRI) study reported an inverted-U-shaped relationship between arousal and saliency-executive network cohesion (Young et al., 2017), which is indicative of a suboptimal engagement of executive control network under high arousal. Congruent with this finding, we here report that participants who experienced high arousal under threat-of-shock demonstrated significantly increased discordance between implicit-explicit cue knowledge, indicating a possible shift to habitual decision-making during the TS phase. Hence, the invertedU relationship between arousal and concordance measure, may suggest that moderate level of arousal or anxiety can aid participants to form more concrete, declarative insights into their implicit strategy over time.

Shifting from goal-directed to habitual decision-making can serve as an adaptive coping mechanism in response to the sustained stressor. Compared to goal-directed behavior, habitual processes require less cognitive resources and thus, lead to faster and more efficient decisions, all of which could help maintain performance (Schwabe \& Wolf, 2013). Additionally, in some 
cases, anxiety can facilitate information processing by reducing interference from task-irrelevant cues (e.g., Easterbrook, 1959; Hu, Bauer, Padmala, \& Pessoa, 2012). In line with this notion, accuracies in a dual-target visual search task, performed under neutral and threat-of-shock conditions, were comparable for high-salience targets, whereas detection of a second, lowsaliency target was impaired under threat-of-shock (Cain et al., 2011). Hence, to cope with anxiety, healthy participants might employ heuristics by selectively focusing on high-value information while ignoring low-valued cues. Many studies have demonstrated that, if employed appropriately, such heuristic models could perform equivalent to or sometimes even better than more complex statistical models (e.g., Czerlinski, Gigerenzer, \& Goldstein, 1999; Martignon, Katsikopoulos, \& Woike, 2008). That is, successful use of satisficing heuristics is heavily dependent on adaptive exploitation of task structure by accurately identifying and utilizing the most important set of information. In our task, we examined post-learning decision behavior once participants had formed a good understanding of the task structure, which might have contributed to the "adaptive" reduction of cue space under threat-of-shock.

Although the threat-of-shock anxiety-induction technique provides an effective way of directly manipulating state anxiety in healthy participants, clinical studies have reported somewhat diverging behavioral effects of pathological anxiety from induced-anxiety (Robinson, Vytal, et al., 2013). That is, patients with clinical anxiety disorders, compared to healthy controls, typically demonstrate elevated baseline arousal (Grillon, Morgan, Davis, \& Southwick, 1998) and exhibit heightened sensitivity to threat-related information (Bar-Haim, Lamy, Pergamin, Bakermans-Kranenburg, \& van IJzendoorn, 2007), which in turn can impair various executive functions such as attentional control (e.g., Martin, Williams, \& Clark, 1991), working memory (e.g., Kizilbash, Vanderploeg, \& Curtiss, 2002), and decision making (e.g., Sailer et al., 2008). 
Therefore, it seems likely that pathological anxiety may interfere with feedback-based learning (Petzold, Plessow, Goschke, \& Kirschbaum, 2010), which could result in inaccurate information usage and inappropriate application of heuristics. Applying our task and methods to clinical populations in future studies may help better understand underlying causes of deficits in learning and decision-making observed in pathological anxiety disorders.

In conclusion, the present study characterized the extent to which anticipatory anxiety induced by threat-of-shock leads to satisficing decision behavior. Throughout the learning and neutral phases, all three groups of participants performed near-optimally, appropriately utilizing all available information to arrive at choices. Under threat-of-shock, however, the high SCL responder group shifted to using the drop-the-worst satisficing strategy by focusing only on a subset of three most informative cues. Additionally, an inverted-U relationship was observed between arousal and implicit-explicit strategy concordance, suggesting that high levels of anxiety increased divergence of participants' actual strategy use from their explicit knowledge. These data have broad implications for understanding and predicting satisficing decision making in high stakes situations, as encountered in medical or military emergencies. 


\section{References}

Bar-Haim, Y., Lamy, D., Pergamin, L., Bakermans-Kranenburg, M. J., \& van IJzendoorn, M. H. (2007). Threat-related attentional bias in anxious and nonanxious individuals: A metaanalytic study. Psychological Bulletin, 133(1), 1-24. http://doi.org/10.1037/00332909.133.1.1

Cain, M. S., Dunsmoor, J. E., LaBar, K. S., \& Mitroff, S. R. (2011). Anticipatory anxiety hinders detection of a second target in dual-target search. Psychological Science: A Journal of the American Psychological Society / APS, 22, 866-871. http://doi.org/10.1177/0956797611412393

Clark, L., Li, R., Wright, C. M., Rome, F., Fairchild, G., Dunn, B. D., \& Aitken, M. R. F. (2012). Risk-avoidant decision making increased by threat of electric shock. Psychophysiology, 49(10), 1436-1443. http://doi.org/10.1111/j.1469-8986.2012.01454.x

Cornwell, B. R., Baas, J. M. P., Johnson, L., Holroyd, T., Carver, F. W., Lissek, S., \& Grillon, C. (2007). Neural responses to auditory stimulus deviance under threat of electric shock revealed by spatially-filtered magnetoencephalography. NeuroImage, 37(1), 282-289. http://doi.org/10.1016/j.neuroimage.2007.04.055

Czerlinski, J., Gigerenzer, G., \& Goldstein, D. G. (1999). How good are simple heuristics? In G. Gigerenzer \& P. M. Todd (Eds.), Simple heuristics that make us smart (pp. 97-118). New York, NY: Oxford University Press.

Drugowitsch, J. (2013). Variational Bayesian inference for linear and logistic regression. arXiv:1310.5438, 1(3), 1-13. 
Dunsmoor, J. E., Mitroff, S. R., \& LaBar, K. S. (2009). Generalization of conditioned fear along a dimension of increasing fear intensity. Learning \& Memory, 16(7), 460-9. http://doi.org/10.1101/1m.1431609

Easterbrook, J. A. (1959). The effect of emotion on cue utilization and the organization of behavior. Psychological Review, 66(3), 183-201. http://doi.org/10.1037/h0047707

Eysenck, M. W., Derakshan, N., Santos, R., \& Calvo, M. G. (2007). Anxiety and cognitive performance: Attentional control theory. Emotion, 7(2), 336-353. http://doi.org/10.1037/1528-3542.7.2.336

Foerde, K., Knowlton, B. J., \& Poldrack, R. A. (2006). Modulation of competing memory systems by distraction. Proceedings of the National Academy of Sciences of the United States of America, 103(31), 11778-83. http://doi.org/10.1073/pnas.0602659103

Gigerenzer, G., \& Gaissmaier, W. (2011). Heuristic decision making. Annual Review of Psychology, 62, 451-82. http://doi.org/10.1146/annurev-psych-120709-145346

Gigerenzer, G., \& Goldstein, D. G. (1996). Reasoning the fast and frugal way: models of bounded rationality. Psychological Review, 103(4), 650-69.

Green, S. R., Kragel, P. A., Fecteau, M. E., \& LaBar, K. S. (2014). Development and validation of an unsupervised scoring system (Autonomate) for skin conductance response analysis. International Journal of Psychophysiology, 91(3), 186-193. http://doi.org/10.1016/j.ijpsycho.2013.10.015

Grillon, C., \& Ameli, R. (1998). Effects of threat of shock, shock electrode placement and darkness on startle. International Journal of Psychophysiology, 28(3), 223-231. http://doi.org/10.1016/S0167-8760(97)00072-X 
Grillon, C., Morgan, C. A., Davis, M., \& Southwick, S. M. (1998). Effect of darkness on acoustic startle in Vietnam veterans with PTSD. American Journal of Psychiatry, 155(6), 812-817. http://doi.org/10.1176/ajp.155.6.812

Grupe, D. W., \& Nitschke, J. B. (2013). Uncertainty and anticipation in anxiety: an integrated neurobiological and psychological perspective. Nature Reviews, 14(July), 488-501. http://doi.org/10.1038/nrn3524

Hu, K., Bauer, A., Padmala, S., \& Pessoa, L. (2012). Threat of bodily harm has opposing effects on cognition. Emotion, 12(1), 28-32. http://doi.org/10.1037/a0024345

Keinan, G. (1987). Decision making under stress: Scanning of alternatives under controllable and uncontrollable threats. Journal of Personality and Social Psychology, 52(3), 639-644. http://doi.org/10.1037/0022-3514.52.3.639

Kizilbash, A. H., Vanderploeg, R. D., \& Curtiss, G. (2002). The effects of depression and anxiety on memory performance. Archives of Clinical Neuropsychology: The Official Journal of the National Academy of Neuropsychologists, 17(1), 57-67. http://doi.org/10.1016/S0887-6177(00)00101-3

Knowlton, B., Mangels, J., \& Squire, L. (1996). A neostriatal habit learning system in humans. Science, 273(5280), 1399-402.

Lykken, D. T., \& Venables, P. H. (1971). Direct measurement of skin conductance: A proposal for standardization. Psychophysiology, 8(5), 656-672. http://doi.org/10.1111/j.14698986.1971.tb00501.x

Martignon, L., Katsikopoulos, K. V., \& Woike, J. K. (2008). Categorization with limited resources: A family of simple heuristics. Journal of Mathematical Psychology, 52(6), 352361. http://doi.org/10.1016/j.jmp.2008.04.003 
Martin, M., Williams, R. M., \& Clark, D. M. (1991). Does anxiety lead to selective processing of threat-related information? Behaviour Research and Therapy, 29(2), 147-160. http://doi.org/10.1016/0005-7967(91)90043-3

Oh, H., Beck, J. M., Zhu, P., Sommer, M. A., Ferrari, S., \& Egner, T. (2016). Satisficing in splitsecond decision making is characterized by strategic cue discounting. Journal of Experimental Psychology: Learning, Memory, and Cognition, 42(12), 1937-1956. http://doi.org/10.1017/CBO9781107415324.004

Otto, A. R., Raio, C. M., Chiang, A., Phelps, E. A., \& Daw, N. D. (2013). Working-memory capacity protects model-based learning from stress. Proceedings of the National Academy of Sciences of the United States of America, 110(52), 20941-20946. http://doi.org/10.1073/pnas.1312011110

Pachur, T., Hertwig, R., \& Wolkewitz, R. (2014). The affect gap in risky choice: Affect-rich outcomes attenuate attention to probability information. Decision, 1(1), 64-78. http://doi.org/10.1037/dec0000006

Petzold, A., Plessow, F., Goschke, T., \& Kirschbaum, C. (2010). Stress reduces use of negative feedback in a feedback-based learning task. Behavioral Neuroscience, 124(2), 248-255. http://doi.org/10.1037/a0018930

Phelps, E. a, Lempert, K. M., \& Sokol-Hessner, P. (2014). Emotion and Decision Making: Multiple Modulatory Neural Circuits. Annual Review of Neuroscience, 263-290. http://doi.org/10.1146/annurev-neuro-071013-014119

Poldrack, R., Clark, J., Paré-Blagoev, E., Shohamy, D., Creso Moyano, J., Myers, C., \& Gluck, M. (2001). Interactive memory systems in the human brain. Nature, 414, 546-550. http://doi.org/10.1038/35107080 
Rhudy, J. L., \& Meagher, M. W. (2000). Fear and anxiety: divergent effects on human pain thresholds. Pain, 84(1), 65-75. http://doi.org/10.1016/S0304-3959(99)00183-9

Rigoux, L., Stephan, K. E., Friston, K. J., \& Daunizeau, J. (2014). Bayesian model selection for group studies - revisited. NeuroImage, 84, 971-85.

http://doi.org/10.1016/j.neuroimage.2013.08.065

Robinson, O. J., Overstreet, C., Charney, D. R., Vytal, K., \& Grillon, C. (2013). Stress increases aversive prediction error signal in the ventral striatum. Proceedings of the National Academy of Sciences of the United States of America, 110(10), 4129-33. http://doi.org/10.1073/pnas.1213923110

Robinson, O. J., Vytal, K., Cornwell, B. R., \& Grillon, C. (2013). The impact of anxiety upon cognition: perspectives from human threat of shock studies. Frontiers in Human Neuroscience, 7(203), 1-21. http://doi.org/10.3389/fnhum.2013.00203

Sailer, U., Robinson, S., Fischmeister, F. P. S., König, D., Oppenauer, C., Lueger-Schuster, B., ... Bauer, H. (2008). Altered reward processing in the nucleus accumbens and mesial prefrontal cortex of patients with posttraumatic stress disorder. Neuropsychologia, 46(11), 2836-2844. http://doi.org/10.1016/j.neuropsychologia.2008.05.022

Schmitz, A., \& Grillon, C. (2012). Assessing fear and anxiety in humans using the threat of predictable and unpredictable aversive events (the NPU-threat test). Nature Protocols, 7(3), 527-32. http://doi.org/10.1038/nprot.2012.001

Schwabe, L., \& Wolf, O. T. (2012). Stress modulates the engagement of multiple memory systems in classification learning. The Journal of Neuroscience, 32(32), 11042-11049. http://doi.org/10.1523/JNEUROSCI.1484-12.2012 
Schwabe, L., \& Wolf, O. T. (2013). Stress and multiple memory systems: From "thinking" to “doing.” Trends in Cognitive Sciences, 17(2), 68. http://doi.org/10.1016/j.tics.2012.12.001

Shackman, A. J., Maxwell, J. S., McMenamin, B. W., Greischar, L. L., \& Davidson, R. J. (2011). Stress Potentiates Early and Attenuates Late Stages of Visual Processing. Journal of Neuroscience, 31(3), 1156-1161. http://doi.org/10.1523/JNEUROSCI.3384-10.2011

Shackman, A. J., Sarinopoulos, I., Maxwell, J. S., Pizzagalli, D. A., Lavric, A., \& Davidson, R. J.

(2006). Anxiety selectively disrupts visuospatial working memory. Emotion, 6(1), 40-61. http://doi.org/10.1037/1528-3542.6.1.40

Simon, H. (1955). A behavioral model of rational choice. The Quarterly Journal of Economics, 69(1), 99-118.

Simon, H. (1990). Invariants of human behavior. Annual Review of Psychology, 41, 1-19. Spielberger, C. D. (1983). Manual for the State-Trait Anxiety Inventory. Palo Alto, CA: Consulting Psychologists Press.

Stephan, K. E., Penny, W. D., Daunizeau, J., Moran, R. J., \& Friston, K. J. (2009). Bayesian model selection for group studies. NeuroImage, 46(4), 1004-17. http://doi.org/10.1016/j.neuroimage.2009.03.025

Thomas, L. a, \& LaBar, K. S. (2008). Fear relevancy, strategy use, and probabilistic learning of cue-outcome associations. Learning \& Memory (Cold Spring Harbor, N.Y.), 15(10), 777784. http://doi.org/10.1101/lm.1048808

Vytal, K. E., Cornwell, B. R., Letkiewicz, A. M., Arkin, N. E., \& Grillon, C. (2013). The complex interaction between anxiety and cognition: insight from spatial and verbal working memory. Frontiers in Human Neuroscience, 7(March), 1-11. 
http://doi.org/10.3389/fnhum.2013.00093

Wang, H., \& Song, M. (2011). Ckmeans.1d.dp: Optimal k-means Clustering in One Dimension by Dynamic Programming. The R Journal, 3, 29-33.

Young, C. B., Raz, G., Everaerd, D., Beckmann, C. F., Tendolkar, I., Hendler, T., ... Hermans, E. J. (2017). Dynamic Shifts in Large-Scale Brain Network Balance As a Function of Arousal.

Journal of Neuroscience, 37(2), 281-290. http://doi.org/10.1523/JNEUROSCI.175916.2017 
Table 1

Cue Weight assignment

\begin{tabular}{cccc}
\hline Cue dimension & Cue state 1 & Cue state 2 & Net cue weight \\
$c_{i}$ & $w 1, i$ & $w 2, i$ & Wnet. $i=w 1, i-w 2, i$ \\
\hline$c_{1}$ & 0.9 & 0.1 & 0.8 \\
$c_{2}$ & 0.8 & 0.2 & 0.6 \\
$c_{3}$ & 0.7 & 0.3 & 0.4 \\
$c_{4}$ & 0.6 & 0.4 & 0.2 \\
\hline
\end{tabular}

Note. Net weights indicate the relative importance of each cue dimension in determining the positive outcome in a given stimulus pair. 
A

A

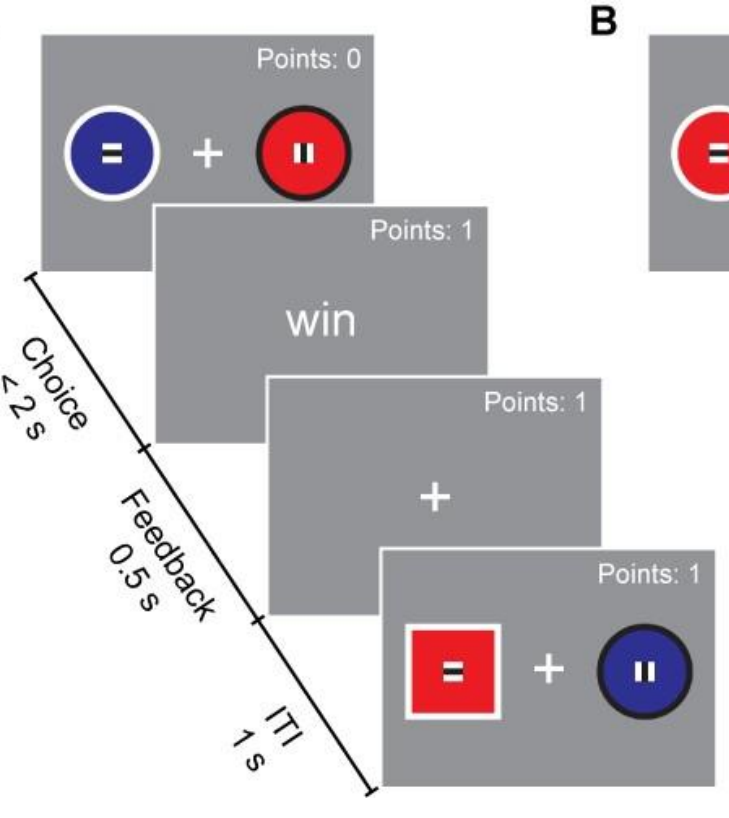

C

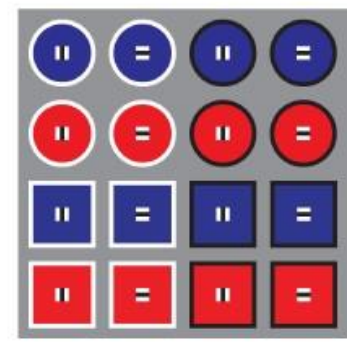

Figure 1. Schematic of the multi-cue probabilistic task. (A) Participants were presented with two different compound stimuli and were asked to predict a stimulus that is more likely to win. Upon each choice, a probabilistic outcome ("win" or "lose") was presented on the screen. Participants earned 1 point for every winning trial. (B) In the threat-of-shock phase, participants additionally experienced randomly occurring shocks at the time of feedback along with a shock symbol. (C) A total of 16 compound stimuli were comprised by combining four cue dimensions (color, shape, contour, and line orientation). 
A

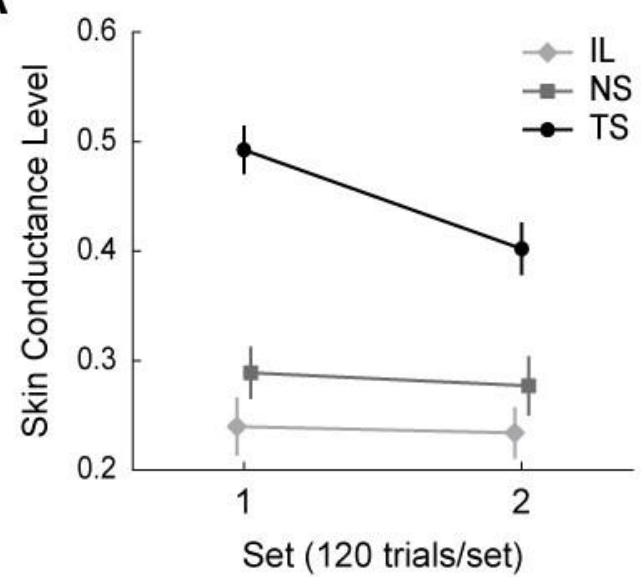

C

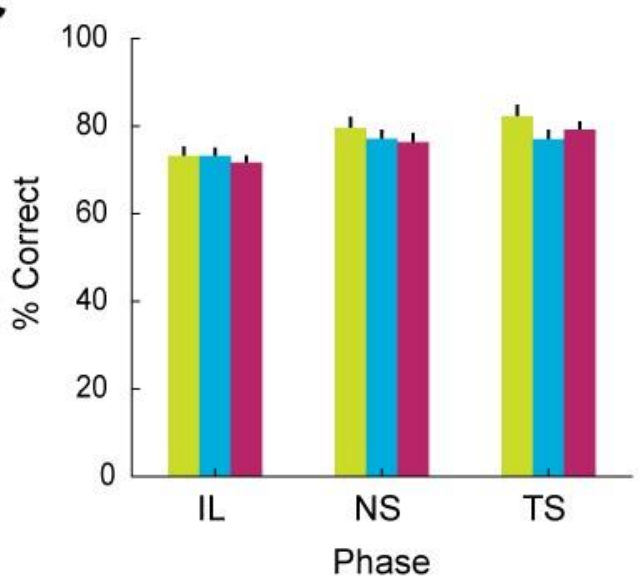

B

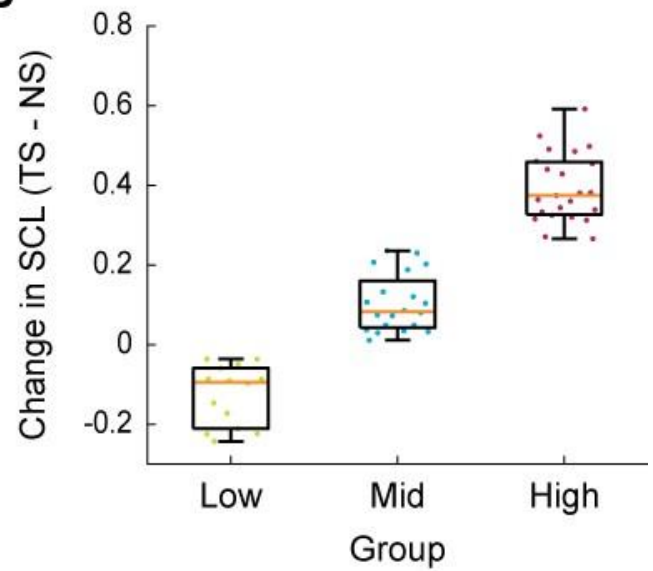

D

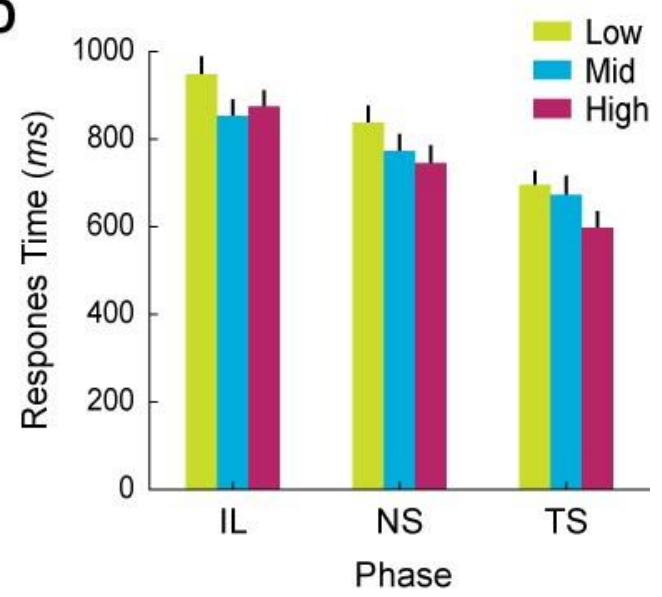

Figure 2. Participant clustering using $\triangle S C L$, and summary of decision performance. (A) Average skin conductance level (range-corrected) per set in each experimental phase. (B) Participant clustering based on changes in SCL $(\triangle S C L)$. Dots represent individual participant. (C) Percent correct choices and (D) mean RT per phase per group. Error bars indicate SEM. 
A

Decision strategy models

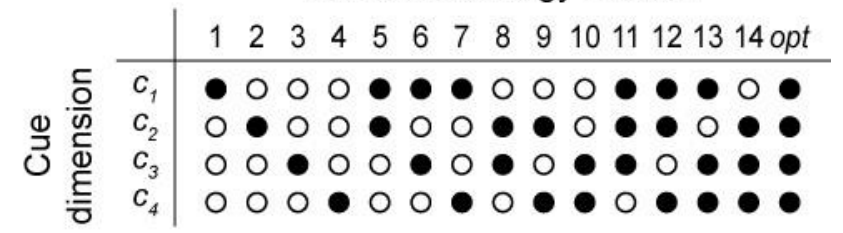

B

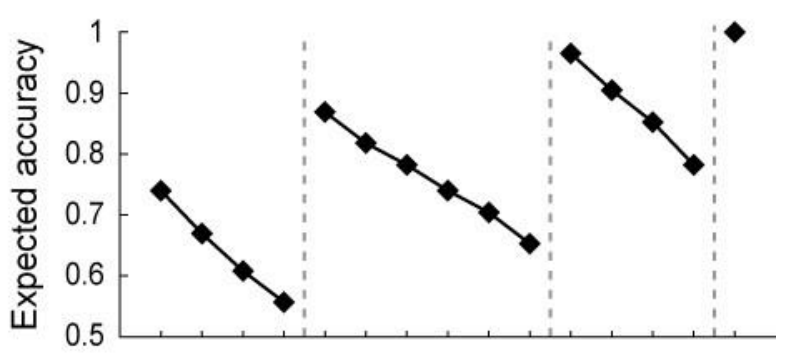

C

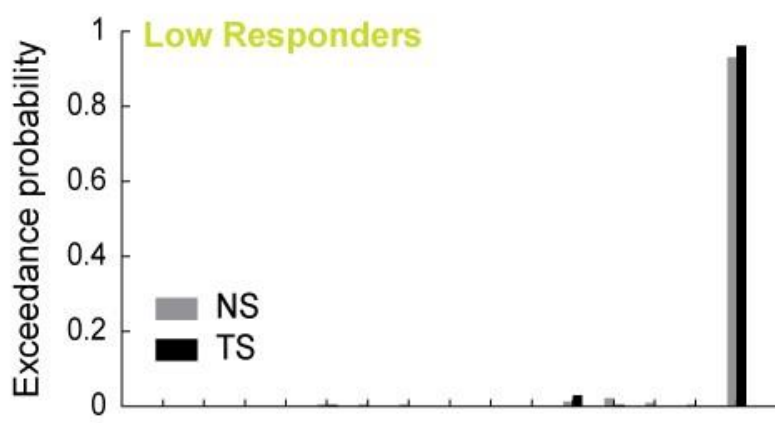

D

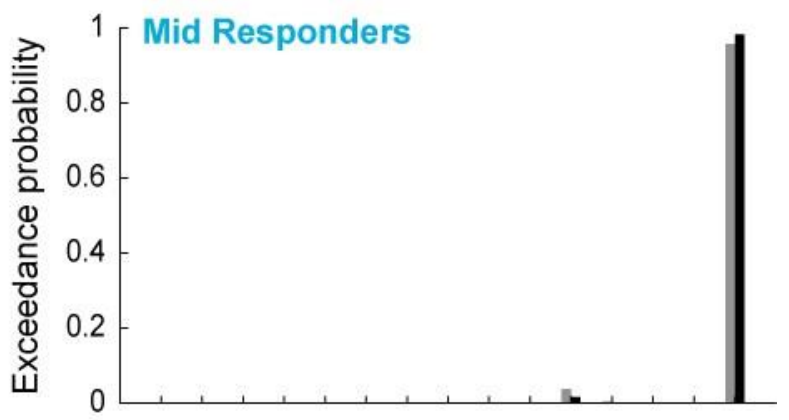

E

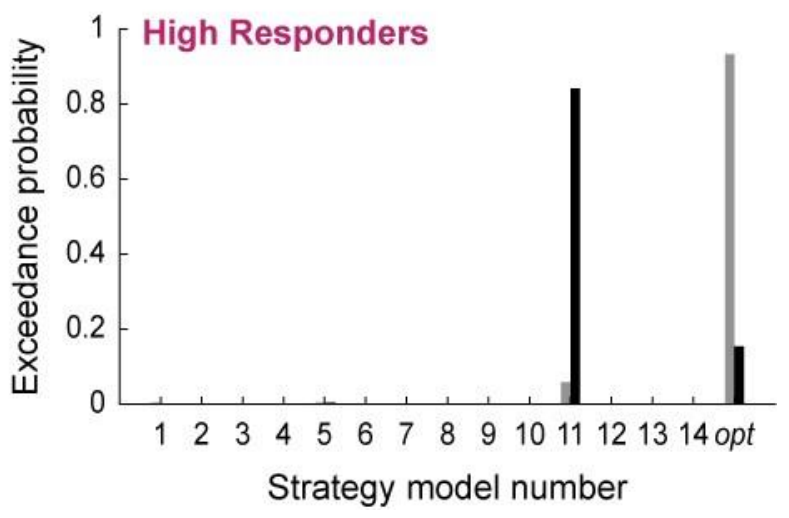

Figure 3. Strategy model selection per responder group. (A) Decision models constructed by combining every possible cue usage. Filled circles $(\bullet)$ denote the cue dimensions that are included in a given model. (B) Expected accuracy for each strategy model estimated based on performance of an ideal observer using a given set of cues. The optimal cue integration model (Model Opt)achieves 100\% accuracy. (C-E) Protected exeedance probabilities during the NS (grey) and TS (black) phase for (C) Low, (D) Mid, and (E) High responder groups. 


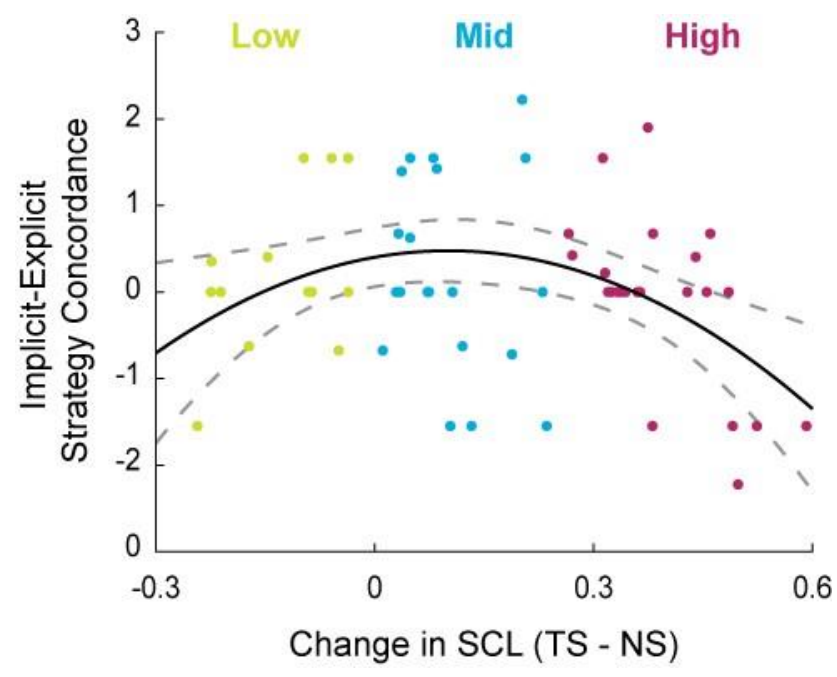

Figure 4. The effect of anticipatory anxiety on task knowledge. A significant inverted-U relationship (solid line, $R_{a d j}{ }^{2}=0.12, p=0.011$ ) was observed between changes in SCL $(\triangle S C L)$ and the implicit-explicit strategy concordance $\left(\Delta r_{S w}\right)$. Colored dots indicate individual participant per group. Dashed lines represent the $95 \%$ CI for the regression line. 